\title{
EDUCAÇÃO ALIMENTAR E NUTRICIONAL PARA O CONTROLE DE COMORBIDADES EM PESSOAS COM DOENÇAS INFECCIOSAS
}

\section{Food and nutrition education for control of comorbidities among people with infectious diseases}

\section{Educación alimentaria y nutricional para el control de comorbidades en personas con enfermedades infecciosas}

\section{Patrícia Dias de Brito}

Instituto Nacional de Infectologia Evandro Chagas - INI/FIOCRUZ - Rio de Janeiro (RJ) - Brasil

\section{Juliana Lauar Gonçalves}

Instituto Nacional de Infectologia Evandro Chagas - INI/FIOCRUZ - Rio de Janeiro (RJ) - Brasil

\section{Paula Simplício da Silva}

Instituto Nacional de Infectologia Evandro Chagas - INI/FIOCRUZ - Rio de Janeiro (RJ) - Brasil

\section{Claudia Santos de Aguiar Cardoso}

Instituto Nacional de Infectologia Evandro Chagas - INI/FIOCRUZ - Rio de Janeiro (RJ) - Brasil

\section{Marlete Pereira da Silva}

Instituto Nacional de Infectologia Evandro Chagas - INI/FIOCRUZ - Rio de Janeiro (RJ) - Brasil

\section{Adriana da Costa Bacelo}

Instituto Nacional de Infectologia Evandro Chagas - INI/FIOCRUZ - Rio de Janeiro (RJ) - Brasil

\section{Raquel Espírito Santo}

Instituto Nacional de Infectologia Evandro Chagas - INI/FIOCRUZ - Rio de Janeiro (RJ) - Brasil

\section{Cristiane Fonseca de Almeida}

Instituto Nacional de Infectologia Evandro Chagas - INI/FIOCRUZ - Rio de Janeiro (RJ) - Brasil

\section{RESUMO}

Objetivo: Relatar a experiência da implantação de práticas de educação alimentar e nutricional (EAN) em grupo, para melhoria de adesão às orientações nutricionais, para pessoas com doenças infecciosas. Síntese de dados: Trata-se de um relato de experiência de atividade de educação alimentar e nutricional realizada no período de abril a novembro de 2015, no Instituto Nacional de Infectologia Evandro Chagas (INI/ Fiocruz), Rio de Janeiro. Doze indivíduos portadores de doenças infecciosas e parasitárias, de ambos os sexos, com diagnósticos de excesso de peso e de síndrome metabólica participaram de sete oficinas temáticas mensais. Foram abordados temas relevantes para o tratamento da síndrome metabólica e do excesso de peso por meio de rodas de conversa, dinâmicas e distribuição de folhetos explicativos. Durante as oficinas, foram identificados de forma clara comportamentos alimentares que não correspondiam à orientação nutricional prévia fornecida na consulta individual e que dificultavam o controle das condições clínicas presentes na síndrome metabólica e excesso de peso. Nas oficinas, os participantes consolidaram o conhecimento sobre práticas alimentares saudáveis e, com a troca de experiência, sentiram-se mais seguros e motivados para superarem as dificuldades durante o tratamento nutricional. Conclusão: Observou-se que os participantes consolidaram seus conhecimentos e a autonomia para escolhas alimentares saudáveis e, com a troca de experiência, sentiram-se mais seguros e motivados para superarem as dificuldades durante o tratamento nutricional. Portanto, a implementação da EAN em grupo foi efetiva na melhora da adesão às orientações nutricionais, refletindo em novos relatos de práticas alimentares saudáveis.

Descritores: Educação Alimentar e Nutricional; Assistência Ambulatorial; Comportamento Alimentar.

\section{ABSTRACT}

Objective: To report the experience of implementing Food and Nutrition Education (FNE) Practices in group, for improvement of nutrition counseling acceptance among patients with infectious diseases. Data synthesis: This is an experience report of food and nutrition education activity carried out from April to November 2015 at the Evandro Chagas National Institute of Infectology (INI/Fiocruz). Twelve individuals 
carrying infectious and parasitic diseases, of both genders, diagnosed with overweight and metabolic syndrome, took part in seven monthly thematic workshops. Themes of relevance to the treatment of metabolic syndrome and overweight were approached by means of group chats and dynamics, and explanatory brochure distribution. During the workshops, eating behaviors were clearly identified, which were not in conformity with the nutrition counseling provided in previous individual appointments and hampered the control of the clinical conditions experienced in metabolic syndrome and overweight. In the workshops, the participants consolidated the knowledge of healthy eating habits and, by exchanging experiences, they felt more confident and motivated to overcome the difficulties during nutrition treatment. Conclusion: It was observed that the participants consolidated their knowledge and autonomy for healthy food choices and, with the exchange of experience, they felt more confident and motivated to overcome the difficulties during the nutrition treatment. Therefore, the implementation of FNE in group was effective in improving nutrition counselling acceptance, which brings forward new reports of healthy eating practices.

Descriptors: Food and Nutrition Education; Ambulatory Care; Feeding Behavior.

\section{RESUMEN}

Objetivo: Describir la experiencia de la aplicación de prácticas de educación alimentaria y nutricional (EAN) en grupo para mejorar la adhesión de las orientaciones nutricionales en personas con enfermedades infecciosas. Síntesis de los datos: Se trata de un relato de experiencia de la actividad de educación alimentaria y nutricional realizada en el periodo entre abril y noviembre de 2015, en el Instituto Nacional de Enfermedades Infecciosas Evandro Chagas (INI / Fiocruz), Rio de Janeiro. Doce personas com enfermidades infecciosas y parasitarias de ambos sexos y diagnostico de exceso de peso y del síndrome metabólico participaron en siete talleres temáticos mensuales. Fueron discutidos temas de interés para el tratamiento del síndrome metabólico y el sobrepeso a través de ruedas de conversación, dinámicas y distribución de folletos. Durante los talleres fueron claramente identificadas las conductas alimentarias que no correspondian con la orientación nutricional previa proporcionada en la consulta individual, lo que hace que sea dificil controlar las condiciones clínicas presentes en el síndrome metabólico y el sobrepeso. En los talleres, los participantes consolidaron el conocimiento sobre prácticas saludables de alimentación y con el intercambio de experiencias se sintieron más seguros y motivados para superar las dificultades en el tratamiento nutricional. Conclusión: Hemos observado con la aplicación del EAN en grupo, una mejora efectiva en la adhesión de las orientaciones nutricionales reflejadas en los nuevos informes de prácticas de alimentación saludables. También observamos que la frecuencia y la regularidad de la vigilancia nutricional tienen un papel crucial en el éxito del tratamiento nutricional.

Descriptores: Educación Alimentaria y Nutrición; Atención Ambulatoria; Comportamiento Alimentación.

\section{INTRODUÇÃO}

Ao longo do tempo, a morbimortalidade por doenças infecciosas no mundo variou de forma significativa em função do desenvolvimento de medidas de prevenção e controle. De forma concomitante, o delineamento do atual perfil epidemiológico dessas doenças transmissíveis foi modificado pela rápida urbanização e facilidade de comunicação entre continentes, países e regiões ${ }^{(1)}$.

No entanto, elas representam um contínuo desafio aos programas de prevenção e tratamento, uma vez que a partir da cronificação das mesmas, novas situações clínicas, como comorbidades metabólicas, adquiririam importância para os cuidados dos indivíduos infectados. Dados recentes mostram uma prevalência de síndrome metabólica de 16,7\% a 31,3\% em pacientes infectados pelo $\mathrm{HIV}^{(2)}$, e de $16,8 \%$ em pacientes com doença de Chagas ${ }^{(3)}$, o que já se assemelha muito a prevalência observada na população geral (entre 17 e 46\%) ${ }^{(2)}$. Dessa forma, é necessário investir em estratégias de prevenção e controle das comorbidades que afetam os indivíduos com doenças infecciosas e parasitárias.

Colocando em perspectiva o público alvo deste relato, percebe-se uma tendência, nos últimos anos, ao declínio na taxa de mortalidade dos indivíduos com doenças infecciosas e parasitária em função de ações articuladas pelo Ministério da Saúde quanto ao aumento da disponibilidade e utilização de instrumentos eficazes de vigilância, prevenção e controle dessas doenças. Entretanto, a tendência para a morbidade nessa população, embora também decrescente, não apresenta a mesma intensidade observada na mortalidade ${ }^{(1)}$.

Tomando como exemplo a doença de Chagas, passada a fase aguda, o indivíduo pode evoluir para uma das formas crônicas, apresentando alterações cardíacas, como insuficiência cardíaca e acidentes tromboembolíticos, e/ou alterações digestivas (megaesôfago e megacólon) ${ }^{(1)}$. No caso da AIDS, a disponibilidade de novas drogas antirretrovirais tem propiciado o aumento da sobrevida das pessoas vivendo com HIV, mas também traz efeitos colaterais que impactam na morbidade, como sobrepeso, dislipidemias e resistência insulínica ${ }^{(1,4)}$. Desta forma, indivíduos com doenças infecciosas e parasitárias podem se tornar doentes crônicos e apresentar condições metabólicas desfavoráveis, aumentando a morbidade de sua doença de base. Dentre as comorbidades mais frequentes ressalta-se a síndrome metabólica e o excesso de peso (sobrepeso e obesidade) ${ }^{(2,3)}$.

A síndrome metabólica associa fatores de risco cardiovasculares, como hipertensão arterial, hipercolesterolemia e diabetes, além da deposição central de gordura, sendo considerada um grande desafio da prática clínica, pois provoca aumento da mortalidade por causas cardiovasculares ${ }^{(5)}$. 
Já o sobrepeso e a obesidade são definidos como um excesso de gordura corporal acumulada no tecido adiposo com implicações para a saúde ${ }^{(6)}$. No Brasil, de acordo com dados da Pesquisa de Orçamentos Familiares (POF) 2008-2009, realizada pelo Instituto Brasileiro de Geografia e Estatística (IBGE) em parceria com o Ministério da Saúde, evidenciou-se um aumento contínuo de excesso de peso na população acima de 20 anos desde 1974. A obesidade cresceu mais de quatro vezes entre os homens, de $2,8 \%$ para $12,4 \%$, e mais de duas vezes entre as mulheres, de $8 \%$ para $16,9 \%{ }^{(7)}$.

As doenças cardiovasculares são tomadas como a maior causa de morte associada ao excesso de peso, porém sabe-se também que pessoas obesas frequentemente desenvolvem outras condições que as predispõem à mortalidade, em especial diabetes, hipertensão, neoplasia, doenças do trato digestivo e esteatose hepática ${ }^{(8)}$.

Diante desse panorama, a educação alimentar e nutricional (EAN) torna-se uma prática fundamental no que concerne à promoção da saúde nesse público. No Brasil, ao longo dos últimos anos, foram conseguidos avanços legais importantes como a Lei Orgânica de Segurança Alimentar e Nutricional (LOSAN) e o Decreto $n^{\circ} 7.272 / 2010$, que cria o Sistema Nacional de Segurança Alimentar e Nutricional, que juntos colocaram a EAN como uma das diretrizes da Política Nacional de Segurança Alimentar e Nutricional (PNSAN). A EAN deve "estimular a autonomia do sujeito para práticas alimentares saudáveis, valorizando e respeitando as especificidades culturais e regionais dos diferentes grupos e etnias, na perspectiva da Segurança Alimentar e Nutricional e da garantia do Direito Humano à Alimentação Adequada "(9).

O comportamento alimentar adequado e o controle do peso corporal são primordiais para diminuir os riscos de agravos à saúde e melhorar o perfil nutricional e metabólico. Nas últimas décadas houve uma modificação do comportamento alimentar na população brasileira, que adotou uma nutrição com alta densidade calórica, excesso de gordura saturada, açúcar simples e sódio, além da ingestão insuficiente de fibras, vitaminas e minerais ${ }^{(10)}$.

Para o manejo dos atuais problemas alimentares e nutricionais vividos pela população, o atendimento nutricional individual em ambulatório desempenha papel estratégico. No entanto, existem fatores multicausais que interferem na eficácia do tratamento nutricional realizado em ambulatório, dentre eles a automotivação para transformações comportamentais que favoreçam práticas alimentares saudáveis.

Neste sentido, o processo da EAN considera que, apesar de serem fatores modificáveis, "as mudanças alimentares voluntárias dependem de um empenho pessoal e de esforço contínuo sobre situações diversas, muitas vezes na contramão de facilidades e mesmo de outras demandas de ordem pessoal e social" ${ }^{\prime(9)}$. Outros aspectos fundamentais são a regularidade e a frequência dos indivíduos às consultas de nutrição. A adesão ao tratamento nutricional está diretamente relacionada ao número de consultas frequentadas ${ }^{(11)}$.

Diante dessas limitações é importante buscar estratégias para aumentar a adesão dos indivíduos às orientações nutricionais, e, entre elas, as práticas de educação e de motivação em grupo, com interações interpessoais, parecem efetivas ${ }^{(12)}$.

No Brasil, alguns autores relatam sua experiência com atividades em grupo como ferramentas úteis para o desenvolvimento de hábitos de vida saudáveis, especialmente àqueles ligados à alimentação, como a EAN ${ }^{(13-20)}$. Neste sentido, a realização das oficinas temáticas em grupo, com encontros mensais, foi uma estratégia de EAN, que se proprôs a aumentar a regularidade e frequência às consultas de nutrição (por reduzir o tempo para a marcação de consultas) e consequentemente tornar mais eficiente o tratamento nutricional.

Portanto, o presente artigo tem como principal objetivo relatar a experiência da implantação de práticas de educação alimentar e nutricional (EAN) em grupo, para melhoria de adesão às orientações nutricionais, para pessoas com doenças infecciosas.

\section{SÍNTESE DOS DADOS}

Trata-se de um relato de experiência realizada no período abril a novembro de 2015, no Instituto Nacional de Infectologia Evandro Chagas (INI), uma unidade da Fiocruz que realiza assistência e pesquisa clínica em doenças infecciosas, no Rio de Janeiro. Nele são atendidos pacientes com HIV/AIDS, infecção pelo HTLV (vírus T-linfotrópico humano), doença de Chagas, leishmaniose, tuberculose, doenças febris agudas, dentre outras.

A identificação dos pacientes aconteceu nos quatro meses anteriores (dezembro de 2014 a março de 2015). Estes pacientes foram triados durante as consultas ambulatoriais individuais de Nutrição, sendo verificado o interesse e disponibilidade em participar do grupo. O foco terapêutico foi o controle das morbidades associadas ao excesso de peso e síndrome metabólica.

Convidaram-se 23 pacientes adultos e idosos, portadores de doenças infecciosas e parasitárias, de ambos os sexos, com diagnóstico de excesso de peso (sobrepeso e obesidade) e de síndrome metabólica. Inicialmente, dezessete aceitaram o convite e afirmaram ter disponibilidade de tempo para participar do programa de EAN. Destes, cinco não compareceram às atividades. Esses pacientes foram contactados por telefone e justificaram sua impossibilidade de frequentar as atividades de EAN por existência de outros compromissos e responsabilidades, como trabalho e marcação de consultas em outras datas, o que aumenta muito a frequência de comparecimento ao hospital, e dificuldades com transporte.

Os pacientes que não aderiram às atividades de EAN em grupo continuaram com o atendimento nutricional individual, com consultas a cada três ou quatro meses. Dessa forma, frequentaram as oficinas 12 pacientes. 
Para o diagnóstico de excesso de peso, foi considerado o Índice de Massa Corporal (IMC) registrado na consulta, obtido a partir da proporção entre peso e estatura ${ }^{(21)}$. O diagnóstico de síndrome metabólica foi definido de acordo com a presença de adiposidade abdominal como condição essencial (circunferência de cintura $\geq 94 \mathrm{~cm}$ em homens e $\geq 80 \mathrm{~cm}$ em mulheres) e, no mínimo, dois critérios dentre os seguintes: triglicerídeo sérico $\geq 150 \mathrm{mg} / \mathrm{dL}$ ou tratamento medicamentoso para hipertrigliceridemia; HDL-colesterol $<40 \mathrm{mg} / \mathrm{dL}$ para homens e $<50 \mathrm{mg} / \mathrm{dL}$ para mulheres; pressão arterial sistêmica $\geq$ 130x $85 \mathrm{~mm} \mathrm{Hg}$ ou tratamento medicamentoso para hipertensão; glicemia de jejum $\geq 100 \mathrm{mg} / \mathrm{dL}$ ou tratamento medicamentoso para diabetes ${ }^{(22)}$.

Para a confirmação do agendamento, todos os pacientes foram contatados por telefone e novamente informados do início das atividades em grupo, uma vez que o recrutamento inicial aconteceu com antecedência média de quatro meses.

O ambulatório do Serviço de Nutrição, rotineiramente utilizado para as consultas individuais, foi o local disponibilizado para o funcionamento das atividades de EAN em grupo. São atendidos no ambulatório pacientes adultos e idosos (maiores de 18 anos), encaminhados pelos médicos e demais profissionais de saúde. A rotina do atendimento nutricional ambulatorial inclui realização de anamnese clínica e alimentar e de medidas antropométricas, checagem de exames laboratoriais, planejamento do tratamento nutricional e orientação nutricional ao paciente.

Por uma questão de limitação de espaço físico e na tentativa de contornar a estigmatização das pessoas vivendo com HIV/ AIDS $^{(23,24)}$, foram agendados dois grupos em datas distintas. Para deixar o espaço mais acolhedor e facilitar a interação entre os pacientes e as nutricionistas, as cadeiras eram previamente dispostas em semicírculo. Um computador foi utilizado para reproduzir slides com ilustrações e explicações sobre o tema de cada oficina. A periodicidade mensal foi escolhida na tentativa de viabilizar a adesão dos participantes, pois moravam em bairros distantes da instituição. No momento da captação, quando perguntados sobre esta periodicidade, os pacientes consideravam viável comparecer uma vez por mês.

O tempo previsto para a duração das oficinas temáticas foi de 90 a 120 minutos (com iníco às 13 horas), na tentativa de não se tornar cansativa ou monótona, e também para atender a solicitação dos próprios pacientes para agilizar o retorno para casa.

Foram previstas sete oficinas temáticas, cada uma delas abordando um tema relevante para o controle do excesso de peso e da síndrome metabólica. Na tentativa de tornar as oficinas mais dinâmicas, foram realizadas atividades interativas, rodas de conversa e distribuição de folhetos explicativos que sintetizavam os pontos mais importantes da oficina temática.

Pela disponibilidade de computador na sala do atendimento e limitação do recursos financeiros para compra de materiais educacionais, a apresentação do tema da oficina era apresentada em slides, sempre utilizando muitas figuras e o mínimo de texto.

Todo o material utilizado nas oficinas foi produzido com base nas publicações do Ministério da Saúde, como o Guia alimentar para a população brasileira ${ }^{(25)} \mathrm{e}$ Alimentos regionais brasileiros ${ }^{(26)}$. A equipe do serviço de Nutrição revisou e aprovou o material.

É importante esclarecer que a proposta de trabalhar a EAN em grupo foi uma meta do Serviço de Nutrição em resposta ao aumento da demanda da marcação de consultas no ambulatório, consequência do aumento da prevalência de complicações metabólicas. Percebeu-se que, a partir de 2010, ocorreu um aumento da demanda por marcação de consultas de Nutrição de primeira vez em função da maior complexidade dos casos de pacientes hospitalizados que recebem alta e demandam cuidados nutricionais específicos, como também pelo aumento da prevalência de complicações metabólicas, um reflexo da cronicidade das doenças infecciosa ${ }^{(1)}$.

Com isso, o tempo de espera para marcação de consultas ultrapassou 100 dias ao fim do primeiro semestre de 2011. Com o acréscimo de um turno de atendimento, esse tempo de espera foi reduzido em $50 \%$ ao final do ano seguinte. No entanto, a partir de 2013, houve nova piora do tempo de espera e, ao mesmo tempo, se detectou uma taxa de absenteísmo de $42 \%$. Em uma análise realizada em conjunto com o Serviço de Enfermagem do INI sobre as causas de absenteísmo às consultas individuais, os pacientes relataram: 1) demora na marcação, 2) esquecimento da marcação da consulta, e 3) falta de dinheiro para pagar a passagem.

Ao final do ano de 2014, observou-se a permanência de uma alta taxa de absenteísmo (média de $46 \%$ para consultas de primeira vez e $40 \%$ para consultas de retorno) e média de 103 dias de fila de espera para marcação de primeira consulta. Diante desse quadro, se questionou se a longa espera para consulta de nutrição poderia impactar negativamente na adesão ao tratamento nutricional.

A partir dessa reflexão surgiu a proposta de trabalhar a EAN em grupo, agregando participantes com comorbidades metabólicas em comum (síndrome metabólica e excesso de peso) em encontros mensais. A criação do grupo teve como objetivo principal a melhoria da adesão dos pacientes às orientações nutricionais, como também a redução da taxa de absenteísmo e do tempo para marcação de consultas no ambulatório.

As oficinas foram conduzidas por três nutricionistas, responsáveis pela seleção e inclusão dos pacientes. Em cada reunião eram aferidos peso corporal e circunferência de cintura. Exames laboratoriais (glicemia e lipidograma) foram monitorados durante o período. Durante as oficinas os participantes eram estimulados a fazer perguntas, tirar dúvidas e contar experiências. Ao final, recebiam o folheto explicativo sobre aquele tema. Também era solicitado que eles trouxessem receitas de preparações que fizessem em casa e considerassem saudáveis, valorizando as especificidades culturais de cada participante. 


\section{Oficina 1:}

Realizou-se a apresentação dos nutricionistas, dos participantes e os objetivos das atividades de EAN em grupo. Foi explicada a definição de síndrome metabólica, desconhecida pelos pacientes, mostrando as condições clínicas definidoras e também as disfunções relativas ao excesso de peso. As nutricionistas apresentaram as vantagens das atividades em grupo, favorecendo a motivação e tornando o tratamento mais dinâmico. Na tentativa de estimular a motivação, foi proposta uma meta objetiva de alteração dos parâmetros nutricionais para os participantes - perda de $10 \%$ do peso corporal e de $5 \%$ da circunferência de cintura.

Em uma dinâmica, os pacientes foram perguntados sobre os principais fatores limitantes à adesão às orientações nutricionais. Algumas das limitações citadas (falta de colaboração da família, eventos festivos, falta de recursos financeiros para compra de alimentos saudáveis e minimamente processados) foram discutidas e, neste momento, as nutricionistas aproveitaram para explicar o papel do comportamento na gênese da obesidade ${ }^{(11)}$. Esta oficina inicial também serviu como base para o aprofundamento dos temas seguintes.

\section{Oficina 2:}

Esta oficina teve como tema alimentação saudável, e a proposta foi a realização do teste Como está a sua alimentação?, baseado no documento do Ministério da Saúde ${ }^{(27)}$. Esta estratégia serviu de base para abordar os grupos alimentares, revisando substituições, e apresentar e discutir a composição de um prato saudável. Os participantes foram estimulados a relatar pontos positivos e negativos da sua rotina alimentar durante a dinâmica.

Observou-se que muitos pacientes apresentavam uma rotina alimentar determinada pelos horários de estudo e trabalho dos familiares, e frequentemente baseada em alimentos ultraprocessados (principalmente carnes processadas). Provavelmente, as consultas individualizadas não são eficientes para levantar todas estas informações, e o atendimento em grupo permitiu o posicionamento dos participantes e maior detalhamento do seu hábito alimentar. Alternativas mais saudáveis aos alimentos ultraprocessados foram sugeridas utilizando como base o Guia Alimentar ${ }^{(25)}$.

\section{Oficina 3:}

$\mathrm{Na}$ tentativa de se abordar dois critérios definidores da síndrome metabólica (hipertrigliceridemia e baixo HDL-colesterol sérico $)^{(5,22)}$, a oficina teve como objetivo discutir essas condições e os tipos de gorduras da alimentação. Para isso, foram explicados a origem e os tipos de lipídeos séricos, as causas e consequências da dislipidemia, e os tipos de gorduras dietéticas. Realizou-se uma dinâmica para que os pacientes optassem pela melhor fonte de gorduras, dentre margarina, manteiga e óleos vegetais; e diferentes tipos de carnes. As comparações foram discutidas pelas nutricionistas para esclarecer o papel das gorduras saturadas na dislipidemia e, portanto, na síndrome metabólica ${ }^{(25)}$. Foram dadas dicas de como reduzir a quantidade de gordura na alimentação e como substituir as gorduras do tipo saturada e trans pelas insaturadas (por exemplo: limitando refogados e frituras, excluindo alimentos ultraprocessados e substituindo molhos prontos por molhos caseiros à base de azeite).

\section{Oficina 4:}

O tema da oficina foi açúcar e edulcorantes. A importância do tema se dá pelo papel do açúcar no aumento do peso e do triglicerídeo sérico ${ }^{(5,8,22)}$. Apresentaram-se os diferentes tipos de açúcar e em quais alimentos estão presentes. Também foi explicada a ação da insulina no organismo, pois os participantes tinham uma percepção errônea da insulina como remédio e não um hormônio produzido pelo corpo. Em uma dinâmica, utilizando embalagens e ilustrações, foram mostradas as concentrações de açúcar em alguns alimentos. Na tentativa de reduzir o açúcar da alimentação, percebeu-se a realização de substituições de sobremesas, refrigerantes e sucos por produtos light e dietéticos, consumindo-os diariamente. Portanto, outro objetivo da reunião foi abordar o uso consciente de edulcorantes, os tipos e os limite de ingestão, também utilizando embalagens e ilustrações na tentativa de alertar para o consumo excessivo.

\section{Oficina 5:}

Como o consumo excessivo de sódio é uma das causas do aumento da pressão arterial, contribuindo para o desenvolvimento da síndrome metabólica ${ }^{(5,22)}$, elaborou-se uma oficina especificamente para abordar essa tema, mostrando o teor de sal nos alimentos e os malefícios da ingestão excessiva de sal. Foram apresentadas ilustrações e embalagens de alimentos industrializados para verificação da quantidade de sódio no rótulo nutricional. Os participantes foram orientados e estimulados a fazer uso do sal de ervas (mistura de sal e ervas).

\section{Oficina 6:}

Esta oficina teve por objetivo apresentar a fibra alimentar. O tema foi escolhido em decorrência do conhecimento consolidado de que a sua ingestão dentro de uma alimentação saudável está relacionada ao controle de níveis de glicose e lipídios séricos ${ }^{(5,8,22)}$. Foi realizada uma dinâmica em que os participantes eram solicitados a citar um alimento que acreditassem 
ser fonte de fibra alimentar e qual a importância da fibra para o organismo. Depois, suas fontes alimentares e funções foram apresentadas e discutidas, assim como sua relação com o controle das condições clínicas da síndrome metabólica e do excesso de peso.

\section{Oficina 7:}

Realizada avaliação final de peso e circunferência de cintura, foi feito um breve resumo dos temas apresentados e uma dinâmica, em que os pacientes foram perguntados sobre o que conseguiram modificar na sua rotina alimentar e se alguma nova informação adquirida no grupo foi incorporada ao hábito.

Como atividade final, foi realizada uma confraternização de encerramento, reunindo os dois grupos, com entrega de relatórios individuais (contendo gráficos de evolução de peso corporal, circunferência de cintura, glicemia e lipidograma), além de certificados de participação. As receitas trazidas pelos pacientes foram compiladas em um caderno, ressaltadas as características: pobre em gordura, pobre em sódio, rica em fibras e/ou sobremessa sem açúcar. Algumas das receitas foram preparadas e levadas para degustação, sendo o caderno distribuído aos pacientes. Para finalizar, realizou-se uma roda de conversa sobre a percepção dos pacientes ao tratamento em grupo.

As atividades em grupo são usadas como ferramentas úteis de EAN e mostram boa resposta no desenvolvimento de hábitos de vida saudáveis, especialmente ligados à alimentação. Entretanto, existem poucos relatos dessas experiências positivas na literatura $^{(13-20)}$. De acordo com o relatório do Encontro de Educação Alimentar e Nutricional - Discutindo Diretrizes ${ }^{(9)}$, uma das principais deficiências dos programas de EAN é a falta de divulgação de resultados bem sucedidos. Portanto, o presente trabalho pretendeu relatar esta experiência de implantação na tentativa de colaborar com a escassa literatura, incentivando outros grupos de profissionais na adoção de uma estratégia conjunta para o desenvolvimento de hábitos alimentares saudáveis.

Observou-se que os pacientes que aceitaram participar e concluíram as atividades eram aposentados ou não trabalhavam, portanto, o que poderia inicialmente ser um complicador por limitar sua situação econômica, favoreceu a adesão ao grupo. Um fato não esperado foi que a frequência nas oficinas foi menor entre os participantes com HIV/AIDS. Acredita-se que um dos motivos seja a estigmatização da doença ${ }^{(23,24)}$ e que esses indivíduos tenham se sentido inseguros quanto à quebra do sigilo de seu diagnóstico em algum momento de discussão das condições de saúde durante as atividades, mesmo tendo sido avisados de que seria um grupo formado apenas por pessoas vivendo com HIV/AIDS.

Nas primeiras oficinas, os participantes tinham receio e vergonha de se expressar, mas, à medida que foram se conhecendo, sentiram-se à vontade para perguntar e contar suas experiências, algumas vezes até saindo do foco do tema da reunião, o que era solucionado com a intervenção de uma das nutricionistas. Como todos os participantes já tinham tido pelo menos uma consulta ambulatorial de Nutrição, o fato da reunião ser conduzida pelas nutricionistas que já faziam o atendimento facilitou a interação.

Durante as atividades, foram identificados comportamentos que não correspondiam à orientação nutricional fornecida anteriormente (na consulta individual) e que dificultavam o controle das condições clínicas presentes na síndrome metabólica e no excesso de peso, como longos períodos de jejum, hábito de beliscar, alta frequência de refeições fora de casa, substituição de refeição (principalmente o jantar) por lanche calórico, excesso de consumo de alimentos industrializados, excesso de café adoçado com açúcar. Dentre os fatores limitantes à adesão das orientações nutricionais citadas, ressaltam-se: hábitos alimentares e rotina dos familiares, horário de trabalho e falta de empenho. Essas limitações à hábitos alimentares saudáveis também são descritas em outros estudos semelhantes ${ }^{(13-20)}$.

Observou-se o relato de hábitos que nunca foram mencionados nas consultas individuais, como consumo frequente de bebida alcóolica e compulsão alimentar. Por outro lado, também foi interessante observar uma visão muito limitada do que seria uma alimentação saudável, além de cobrança em relação a restrições alimentares desnecessárias e desmotivadoras, já que não eram cumpridas, mas que eles acreditavam que seriam defendidas pelas nutricionistas.

Modificações comportamentais durante as refeições também foram abordadas nas dinâmicas do grupo, incluindo a adequada mastigação e deglutição. Entretanto, observou-se que nem sempre o local de refeições era um ambiente calmo, que propiciasse a adequada mastigação, e frequentemente ocorria a ingestão concomitante de grandes volumes de líquido durante as refeições.

Ao longo das oficinas, o momento da pesagem e da medida da circunferência de cintura trazia muita expectativa para alguns participantes, então se passou a ressaltar o caráter puramente estimulatório da meta e que, se não atingida, não prejudicaria a participação nas atividades.

$\mathrm{Na}$ dinâmica da avaliação final, os participantes expressaram sua opinião sobre as práticas de EAN em grupo. Eles se mostraram satisfeitos com a proposta, os temas abordados e a periodicidade. Os participantes que não atingiram a meta justificaram o insucesso com a falta de comprometimento próprio e solicitaram participar novamente do grupo. Desta forma, se estabeleceu que aqueles que tiveram $75 \%$ de presença e/ou atingiram a meta proposta, passariam para um grupo de manutenção, com encontros a cada 4 meses. Os demais poderiam participar novamente do grupo no ano de 2016.

Pôde-se identificar alguns fatores limitantes durante a implantação dessa proposta de EAN, como a falta de recursos financeiros para compra de materiais educacionais. O espaço físico foi adaptado para a realização das oficinas, mas não era adequado à atividades mais interativas. Os pacientes consideraram a periodicidade e o tempo de duração das oficinas adequados, mas, com relação ao turno das atividades (realizadas a tarde), relataram preferir comparecer ao hospital no turno da manhã. 
Com bases nesses fatores limitantes foram estabelecidas algumas modificações para o grupo de 2016, como atividades mais dinâmicas e o foco na presença às reuniões e nas modificações de comportamento alimentar, deixando as alterações dos parâmetros nutricionais como metas secundárias. No entanto, não houve mudança do local por falta de espaço físico mais adequado e também não houve alteração para o turno da manhã por indisponibilidade de sala.

Em contrapartida, a experiência apresentou vários aspectos positivos. O momento do atendimento em grupo foi relatado com um espaço onde os participantes puderam expressar livremente sua opinião e confrontar suas ideias, o que normalmente não acontece nas consultas individuais. A partir disso, a equipe apreendeu sobre a importância do vínculo profissional-paciente, e que a comunicação acessível garante um diálogo efetivo, evitando distorções do compreendimento das orientações dietéticas.

Além disso, ressalta-se a facilidade de aceitação da proposta pela instituição, que vem garantindo a manutenção da atividade como parte da assistência nutricional. A proposta implantada teve como desdobramento o desenvolvimento de um projeto de pesquisa, no qual serão inseridos discentes do curso de especialização de Nutrição Clínica aplicada à Infectologia.

Ao final do primeiro trimestre de 2016, o tempo de espera para marcação de consultas reduziu em $60 \%$, o que se acredita ter sido, pelo menos em parte, devido à implantação das atividades de EAN em grupo com foco no tratamento do excesso de peso e da síndrome metabólica.

A partir dessa experiência acredita-se que a EAN em grupo seja uma eficiente estratégia de atenção à saúde, que pode ser implementada para otimizar o cuidado nutricional nas comorbidades metabólicas de pacientes com doenças infecciosas e parasitárias. No entanto, são necessários trabalhos futuros para avaliar a efetividade dessas práticas de EAN na melhora dos parâmetros antropométricos e clínicos dos pacientes.

\section{CONCLUSÃO}

Observou-se que os participantes consolidaram seus conhecimentos e a autonomia para escolhas alimentares saudáveis e, com a troca de experiências, sentiram-se mais seguros e motivados em superar as dificuldades durante o tratamento nutricional. Portanto, a implementação da EAN em grupo foi efetiva na melhora da adesão às orientações nutricionais, refletindo em novos relatos de práticas alimentares saudáveis.

\section{AGRADECIMENTOS}

Ao Eric Henrique Roma de Lima pela revisão da língua inglesa e à Sabrina Rose Hermann pela tradução para o espanhol.

\section{CONFLITOS DE INTERESSE}

Os autores deste trabalho não possuem conflitos de interesse para declarar.

\section{REFERÊNCIAS}

1. Ministério da Saúde (BR). Doenças infecciosas e parasitárias: guia de bolso. $8^{\mathrm{a}}$ ed. rev. Brasília: Ministério da Saúde; 2010.

2. Nguyen KA, Peer N, Mills EJ, Kengne AP. A meta-analysis of the metabolic syndrome prevalence in the global HIVinfected population. PLoS ONE. 2016;11(3):e0150970.

3. Jackson Y, Castillo S, Hammond P, Besson M, Brawand-Bron A, Urzola D, et al. Metabolic, mental health, behavioural and socioeconomic characteristics of migrants with Chagas disease in a non-endemic country. Trop Med Int Health. 2012;17(5):595-603.

4. Ministério da Saúde (BR). Protocolo clínico e diretrizes terapêuticas para manejo da infecção pelo HIV em adultos. Brasília: Ministério da Saúde; 2013.

5. Sociedade Brasileira de Hipertensão, Sociedade Brasileira de Cardiologia, Sociedade Brasileira de Endocrinologia e Metabologia, Sociedade Brasileira de Diabetes, Associação Brasileira para Estudos da Obesidade. I Diretriz Brasileira de Diagnóstico e Tratamento da Síndrome Metabólica. Arq Bras Cardiol. 2005;84(1):3-28.

6. World Health Organization - WHO. Reducing risks, promoting healthy life. Geneva: WHO; 2002.

7. Instituto Brasileiro de Geografia e Estatística - IBGE, Ministério do Planejamento, Orçamento e Gestão. Pesquisa de Orçamentos Familiares 2008-2009 [Internet]. Rio de Janeiro: IBGE; 2010 [acesso em 2016 Jun 10]. Disponivel em: http//: www.ibge.gov.br

8. Jung RT. Obesity as a disease. Brit Med Bull. 1997;53(2):307-21. 
9. Ministério da Saúde (BR), Coordenação-Geral de Educação Alimentar e Nutricional. Relatório final do Encontro de Educação Alimentar e Nutricional: discutindo diretrizes. Brasília: Ministério da Saúde; 2011.

10. Levy RB, Claro RM, Mondini L, Sichieri R, Monteiro CA. Distribuição regional e socioeconômica da disponibilidade domiciliar de alimentos no Brasil em 2008-2009. Rev Saúde Pública. 2012;46 (1):6-15.

11. Janda M, Zeidler D, Bohm G, Schoberberger R. An instrument to measure adherence to weight loss programs: the Compliance Praxis Survey-Diet (COMPASS-Diet). Nutrients. 2013;5(10):3828-38.

12. Chimenti BM, Bruno MLM, Nakasato M, Isosaki M. Estudo sobre adesão: fatores intervenientes na dieta hipocalórica de coronariopatas internados em um hospital público de São Paulo. Rev Bras Nutr Clin. 2006;21(3):204-10.

13. Meirelles ARN, Luedy A, Menezes D, Ribeiro H. Implantação de um programa de educação do paciente em um hospital público. Rev Baiana Saúde Pública. 2015;39(3):668-80.

14. Pereira MA, Pereira AA, Leão JM, Lisboa LCV, Elias MAR, Ghetti FF, et al. Desafios e reflexões na implantação de um programa de educação alimentar e nutricional (EAN) em indivíduos com excesso de peso. Rev Bras Promoç Saúde. 2015;28(2):290-6.

15. Franzoni B, Lima LA, Castoldi L, Labrêa MGA. Avaliação da efetividade na mudança de hábitos com intervenção nutricional em grupo. Ciênc Saúde Coletiva. 2013;18(12):3751-8.

16. Moreira P, Romualdo MCS, Amparo FC, Paiva C, Alves R, Magnoni D, et al. A educação nutricional em grupo e sua efetividade no tratamento de pacientes obesos. RBONE. 2012;6(35):216-24.

17. Ferreira FB, Fraga JCS, Nunes JP, Liberali R, Navarro F. Alterações antropométricas de pacientes obesos submetidos a um tratamento multidisciplinar da obesidade em Porto Alegre. RBONE. 2009;3(16):290-7.

18. Dias LCGD, Fioravante M, Zacarin JF, Lopes TVC. Reeducação alimentar no programa de saúde da família: relato de experiência. Rev Ciênc Ext. 2008;4(1):122-8.

19. Maffacciolli R, Lopes MJM. Educação em Saúde: a orientação alimentar através de atividades de grupo. Acta Paul Enferm. 2005;18(4):439-45.

20. Diogo MJDE, Ceolim MF, Cintra FA. Implantação do grupo de atenção à saúde do idoso (GRASI) no Hospital de Clínicas da Universidade Estadual de Campinas (SP): relato de experiência. Rev Latinoam Enferm. 2000;8(5):85-90.

21. World Health Organization - WHO. Obesity: preventing and managing the global epidemic. Report of a WHO Consultation. Geneva: WHO; 2000. (Technical Report Series 894).

22. Sociedade Brasileira de Cardiologia. V Diretriz Brasileira sobre Dislipidemia e Diretrizes de Prevenção da Aterosclerose do Departamento de Aterosclerose da SBC. Arq Bras Cardiol. 2013;101(4 Supl 1):1-22.

23. Garcia S, Koyama MAH. Estigma, discriminação e HIV/Aids no contexto brasileiro, 1998 e 2005 . Rev Saúde Pública. 2008;42(Supl 1):72-83.

24. Parker R, Agleton P. Estigma, discriminação e AIDS. Rio de Janeiro: Associação Brasileira Interdisciplinar de AIDS; 2001. (Coleção ABIA, Cidadania e Direitos, 1).

25. Ministério da Saúde (BR), Secretaria de Atenção à Saúde, Departamento de Atenção Básica. Guia alimentar para a população brasileira. $2^{\mathrm{a}}$ ed. Brasília: Ministério da Saúde; 2014.

26. Ministério da Saúde (BR), Secretaria de Atenção à Saúde. Departamento de Atenção Básica. Alimentos regionais brasileiros. $2^{\mathrm{a}}$ ed. Brasília: Ministério da Saúde; 2015.

27. Ministério da Saúde (BR), Secretaria de Atenção à Saúde, Coordenação Geral da Política de Alimentação e Nutrição. Guia alimentar: como ter uma alimentação saudável. Brasília: Ministério da Saúde; [s.d.]. (Guia de bolso).

\section{Endereço para correspondência:}

Patrícia Dias de Brito

Avenida Brasil, 4365

Bairro: Manguinhos

CEP: 21040-900 - Rio de Janeiro -RJ - Brasil

E-mail: didibrito@yahoo.com.br 